\title{
EAR RECOGNITION USING A NEW LOCAL MATCHING APPROACH
}

\author{
Yimo Guo, Zhengguang Xu \\ School of Information Engineering \\ University of Science and Technology Beijing \\ Beijing, 100083, China
}

\begin{abstract}
A new ear recognition approach, including a feature extraction method and the recognition framework, is presented in this paper. The proposed feature extraction method, called the Local Similarity Binary Pattern (LSBP), considers both the connectivity and similarity information in representation. In ear recognition, LSBP is combined with the Local Binary Pattern (LBP) to represent the ear image. The concatenated histogram sequences encode more relationships among neighborhoods that are shown to be discriminative. To enhance efficient representation, Cellular Neural Network is adopted to preprocess images, the function of which is to eliminate irrelevant information. From the experimental results conducted on the USTB ear database, the proposed approach outperforms some other well-known methods in terms of the recognition rate.
\end{abstract}

Index Terms - Pattern recognition, image representations, feature extraction

\section{INTRODUCTION}

Over the last decade, ear has been gradually considered as a biometric identifier. Related research involves 2D ear recognition, 3D ear recognition, ear-print recognition and so on. Although many approaches have been proposed, the 2D ear recognition remains challengeable in real application. Some widely used methods in pattern recognition have been applied to the 2D ear recognition. One of the representative methods is the Principal Component Analysis (PCA) for ear recognition [1]. However, better recognition performance could be achieved under limited conditions because of the inherent shortages of PCA. Linear Discriminant Analysis (LDA) and kernel methods were also introduced. But the problem of generalized eigenvalue with LDA has not been well solved [2]. As most of the previous methods adopted are feature-based (structural) matching methods, the recognition performance is easily affected by irrelevant information [3][4][5]. Besides, the methods based on statistical learning theory may confront the generalization problem.
In pattern recognition, the key task is to explore discriminative information and extract features accordingly. As we know, ear-print is a special and prominent characteristic of the ear. The ear skeleton and ear shape contribute a lot to the recognition, which can be embodied by the connectivity and similarity information. Therefore, we take the connectivity and similarity of intensity into consideration to represent the ear image. Given this representation method, the recognition is local matching rather than statistical learning theory based.

The rest of this paper is organized as follows. In Section 2 , the new feature extraction method for ear recognition is described. In Section 3, we put forward the framework of the ear representation. Experiments are also reported in this section. Conclusions are drawn in Section 4.

\section{EAR REPRESENTATION WITH LOCAL SIMILARITY BINARY PATTERN}

\subsection{Local Similarity Binary Pattern}

Local Binary Pattern is an effective feature extraction method in pattern recognition. The original LBP operator was introduced for texture classification [6] and demonstrated to be a powerful texture descriptor. The operator labels pixels by thresholding $n \times n$ neighborhoods with the center value. The result is recorded as a binary number and the histogram of the labels is considered as a texture descriptor. Later, LBP was extended to face recognition with some modifications [7]. The operator still labels pixels by thresholding $n \times n$ neighborhoods with the center value. The result is recorded as a binary number and the histogram of the uniform patterns, instead of the labels, is considered as a descriptor. As LBP captures the shape and texture information in representation, it is sensitive to high frequency components.

Considering the characteristics of the ear image, the proposed feature extraction method put an emphasis on the connectivity and similarity information. As previous work, we use the notation $(\mathrm{P}, \mathrm{R})$ for neighborhoods. The operator labels pixels by comparing the neighborhoods with the center value. $L(n)$ is the label of the $n$th neighborhood and it follows the rules: 


$$
L(n)=\left\{\begin{array}{cc}
1 & \text { equal or closest values } \\
0 & \text { other values } \\
n=1,2, \ldots P
\end{array}\right.
$$

In detail, the rule of $L(n)=1$ is defined as:

$$
L(n)=1 \Leftrightarrow \begin{cases}g_{n}=g_{0} & \text { equal values } \\ \underset{g_{n} \rightarrow \bar{g}}{\operatorname{Min}}\left|g_{n}-g_{0}\right| & \text { closest values }\end{cases}
$$

Where $g_{n}$ is the value of the $n$th neighborhood, $g_{0}$ is the value of the center pixel and $\bar{g}$ is the mean value of the $n \times n$ neighborhoods. The neighborhoods that have the same value as the center value are labeled to 1 . If there is no such pixels, neighborhoods with the closest value are labeled to 1 . Figure 1 is an illustration of the LSBP operator in this case. The minimum difference between the neighborhoods and the center value is 1, therefore, 6 and 8 are the closer values. To determine the closest value, mean value $\bar{g}$ is calculated and compared with the closer values. Because 6 is closer to $\bar{g}$ than 8 , it is considered to be the closest value and labeled to 1 . This feature extraction method is called the Local Similarity Binary Pattern (LSBP). Figure 2 gives some examples of the circular $(8,1)$ neighborhoods and their corresponding binary numbers. The neighborhoods in gray are equal or closest to the center value. In this way, each pixel is recorded as a binary number and the histogram of the uniform patterns is an ear descriptor.

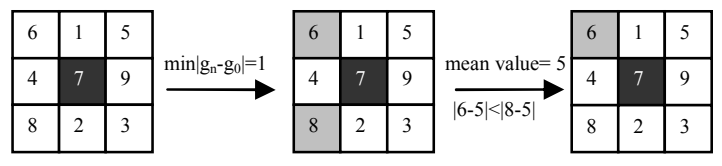

Fig. 1. The LSBP operator

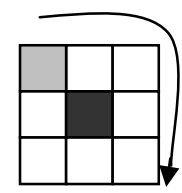

Binary: 10000000

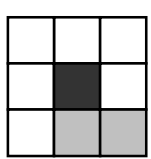

00001100

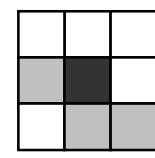

00001101

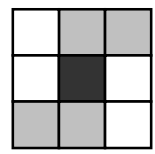

01100110
Fig. 2. Examples of the circular neighborhoods

\subsection{Preprocessing with the Cellular Neural Network}

Different from the texture analysis and fingerprint recognition, to which the local connectivity contributes a lot, holistic connectivity and similarity play a more significant role in ear recognition. Therefore, the Cellular Neural Network (CNN) is utilized to preprocess ear images for holistic feature extraction. The function of the $\mathrm{CNN}$ is to eliminate irrelevant information and retain useful information. It can highlight the ear skeleton and ear shape.
The architecture of the $\mathrm{CNN}$ is a two-dimensional array of cells. A standard $\mathrm{CNN}$ is defined by a $M \times N$ rectangular array of cells $C(i, j)$ that locate at site $(i, j)$, where $i=1,2, \ldots, M ; j=1,2, \ldots, N$. Each cell $C(i, j)$ is defined by the dynamic equation:

$$
\dot{x}_{i, j}=-x_{i, j}+\sum_{k=-1}^{1} \sum_{l=-1}^{1} a_{k, l} y_{i+k, j+l}+\sum_{k=-1}^{1} \sum_{l=-1}^{1} b_{k, l} u_{i+k, j+l}+z
$$

Where $x_{i, j}, y_{i+k, j+l}, u_{i+k, j+l}, z_{i, j}$ are the state, output, input and threshold of the cell respectively. $a_{k, l}(i, j, t)$ and $b_{k, l}(i, j, t)$ are the feedback and input synaptic operators [8]. To realize the expected functions, the Global Task and Local Rules are described as follows.

The Global Task:

1) Given: A static grayscale image $P$.

2) Input: $U(t)=P$.

3) Initial state: $X(0)=$ Arbitrary or $X(0)=0$.

4) Boundary condition: $u_{i, j}=y_{i, j}=0$ for all virtual cells.

5) Output: $Y(t) \Rightarrow Y(\infty)=$ Binary image. Pixels in $P$ become black if they had belonged to some convex objects before the objects were disturbed. Otherwise, pixels become white.

The Local Rules:

$$
\begin{array}{ccc}
u_{i, j}(0) & \rightarrow & y_{i, j}(\infty) \\
u_{i, j}(0)=1 \text { or }-1 & \rightarrow & y_{i, j}(\infty)=\text { white },
\end{array}
$$

If there are at least 5 or more white nearest neighbors.

$$
u_{i, j}(0)=1 \text { or }-1 \quad \rightarrow \quad y_{i, j}(\infty)=\text { black }
$$

If there are at most 4

\begin{tabular}{|c|c|c|c|}
\hline \multirow{3}{*}{$A=$} & 0 & 0 & 0 \\
\hline & 0 & 0 & $\overline{0}$ \\
\hline & 0 & 0 & 0 \\
\hline \multirow{3}{*}{$B=$} & 2 & 2 & 2 \\
\hline & 2 & 0 & 2 \\
\hline & 2 & 2 & 2 \\
\hline
\end{tabular}
white nearest neighbors.

The output $y_{i, j}$ is the piece-wise linear function given by the equation:

$$
\begin{gathered}
y_{i+k, j+l}=\frac{1}{2}\left(\left|x_{i+k, j+l}+1\right|-\left|x_{i+k, j+l}-1\right|\right) \\
i=1,2, \ldots, M ; j=1,2, \ldots, N .
\end{gathered}
$$

The proofed $\mathrm{CNN}$ template has the form: 

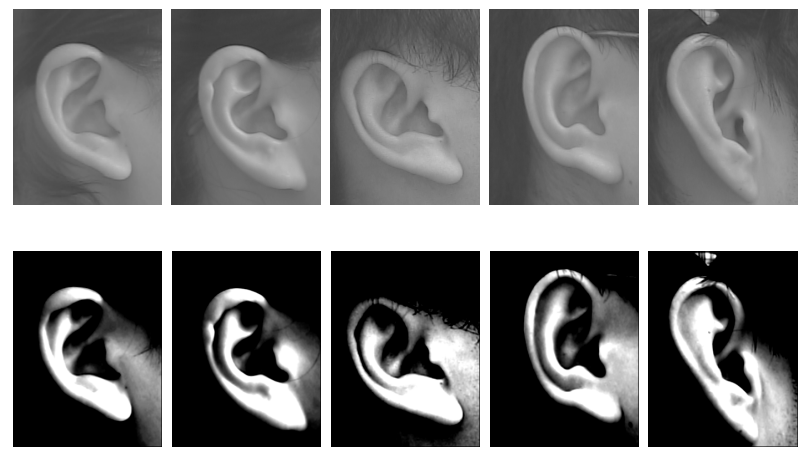

Fig. 3. Samples of the ear images and the processed images

Figure 3 shows some ear images from the database and images processed by the CNN. From the processed images, we can see that some unfavorable information obvious in the original images disappears, but the skeleton and shape are more obvious.

\section{EXPERIMENTS}

LSBP histogram captures the information of connectivity and similarity, such as lines and connective area; while LBP feature vector contains information about distribution, such as edges, spots and flat areas [7]. Considering their superiorities, two features are both adopted in ear recognition. In addition, to enhance efficient representation, histograms not only encode local information but also spatial information by image decomposition. As local matching approaches, LBP and LSBP have relation with the parameters, that is, the feature dimension and recognition rate are dependent on the parameters. Therefore, we analysis the recognition rates for LBP operators as a function of the window numbers. The experiment is conducted on a subset (40 subjects) of the USTB ear database that contains 308 images of 77 subjects [5]. These images are taken under 3 viewing conditions (azimuth $\alpha \in\{-30,0,30\}$ ) and different illumination conditions. The original images, with a resolution of $300 \times 400$, are cropped to grayscale images with a resolution of $270 \times 360$. Samples of two subjects are shown in figure 4. In this experiment, three images of a subject are taken as the training set and the remaining one serves as the testing set. The classifier is the simplest classification scheme: nearest neighbor classifier in the image space, with the Chi square statistics as the similarity measure. The mean recognition rates of the LBP operators $(8,1),(8,2)$ and $(16,2)$ as a function of the window numbers are plotted in figure 5. The images are divided into $k \times k$ windows, $k=1,2, \ldots 8$, which results in the window size from $270 \times 360$ to $34 \times 45$.
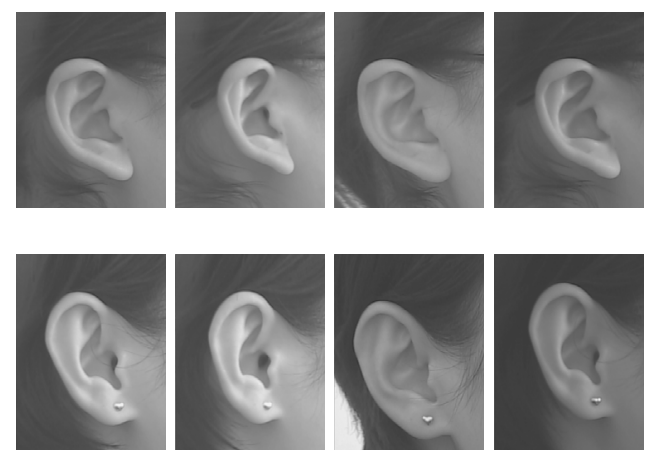

Fig. 4. Sample images of two subjects

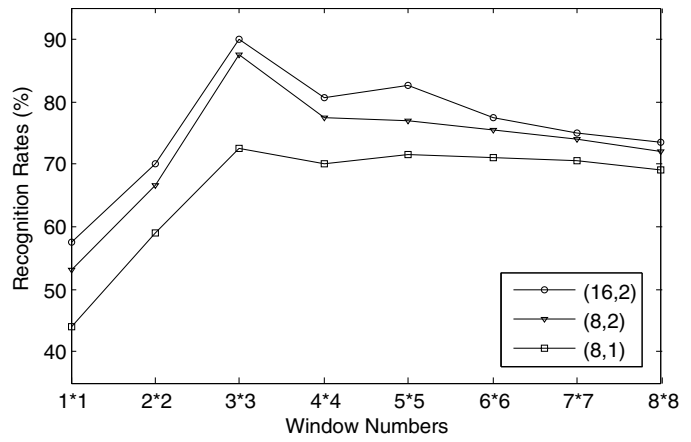

Fig. 5. Recognition rates of three operators as a function of the window numbers

As illustrated in figure 5, the recognition performance can be improved by image decomposition. Therefore, in the experiment, we decompose images into $3 \times 3$ windows and select the operator $(16,2)$. In ear recognition, the original images and processed images are decomposed into regions from which LBP features and LSBP features are extracted respectively. The spatially enhanced histogram is defined as the combination of these two kinds of features. In this way, the textures of ear regions are locally encoded by micropatterns and the shape is recovered by the construction of the ear feature histogram. The framework of the ear image representation is illustrated in figure 6.

To validate the proposed method, it is compared with some other methods: Principal components analysis (PCA) [1], Fisher Discriminant Analysis (FDA) [2], Independent components analysis (ICA) [9], geometric approach [3], rotation invariant descriptor [4], improved non-negative matrix factorization with sparseness constraints (INMFSC) [10] and Local binary pattern [7]. The recognition rates (in $\%)$ are listed in Table 1. In these experiments, the training set consists of three random images from each subject and the remaining images serve as the testing set. 


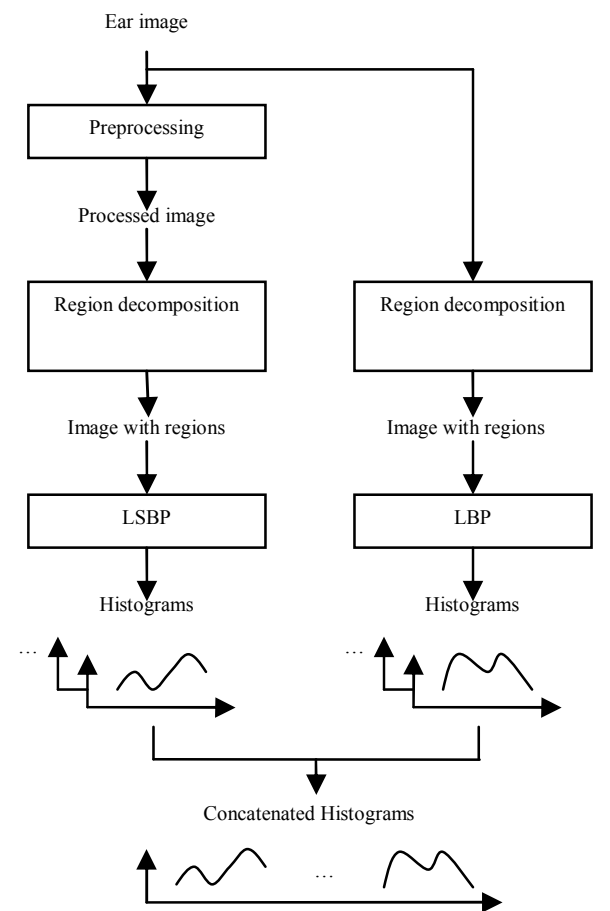

Fig. 6. The framework of the ear representation approach

\begin{tabular}{|c|c|c|c|}
\hline Methods & $\begin{array}{c}\text { Mean } \\
\text { recognition } \\
\text { rate }\end{array}$ & $\begin{array}{c}\text { Upper } \\
\text { recognition } \\
\text { rate }\end{array}$ & $\begin{array}{c}\text { Lower } \\
\text { recognition } \\
\text { rate }\end{array}$ \\
\hline PCA & 78.6 & 81 & 73 \\
\hline ICA & 83.6 & 86.5 & 79 \\
\hline FDA & 85.7 & 88 & 81 \\
\hline $\begin{array}{c}\text { Geometric } \\
\text { approach }\end{array}$ & 87 & 92 & 84.5 \\
\hline INMFSC & 86.6 & 93 & 74 \\
\hline $\begin{array}{c}\text { Rotation invariant } \\
\text { descriptor }\end{array}$ & 88 & 92.5 & 82 \\
\hline LBP & 90 & 93 & 85.5 \\
\hline Our approach & 93.2 & 97.5 & 85 \\
\hline
\end{tabular}

Table 1. Experimental results of the comparison (\%)

\section{CONCLUSIONS}

We present a novel approach, including the feature extraction method and the recognition framework, for ear recognition. As in other biometrics, ear images are considered as a composition of micro-patterns that can be well described by local matching approaches. Because of the special characteristics of ear images, the connectivity and similarity of intensity plays a significant role in recognition, which can be encoded by the proposed Local Similarity Binary Pattern. Benefit from its combination with the LBP, the final representation contains the information of both the distribution and connectivity. Moreover, original images and processed images are decomposed into regions, from which features are extracted. Therefore, the global feature histogram they construct represents not only the statistics of micro-patterns but also the spatial locations. From the experimental results, this approach outperforms some other well-known methods under the variations of view point and illumination.

\section{REFERENCES}

[1] K. Chang, K. Bowyer, S. Sarkar and B. Victor, "Comparison and Combination of Ear and Face Images in Appearance-Based Biometrics," IEEE Transactions on Pattern Analysis and Machine Intelligence, vol. 25, no. 9, pp. 1160-1165, 2003.

[2] Y. Liu, Z Mu and L. Yuan, "Application of Kernel Function Based Fisher Discriminant Analysis Algorithm in Ear Recognition," Measurements and Control, vol. 22, no. 8, pp. 304306, 2006.

[3] D. Shailaja, P. Gupta, "A Simple Geometric Approach for Ear Recognition," The 9th International Conference on Information Technology, Bhubaneswar India, pp. 164-167, 2006.

[4] A. Fabate, M. Nappi, D. Riccio and S. Ricciardi, "Ear Recognition by means of a Rotation Invariant Descriptor," The 18th International Conference on Pattern Recognition, Hong Kong, vol. 4, pp. 437-440, 2006.

[5] L. Yuan, Z. Mu, "Ear Recognition based on 2D Images," The 1th IEEE International Conference on Biometrics: Theory, Applications, and Systems, Washington DC, pp. 1-5, 2007. (http://www.ustb.edu.cn/resb/)

[6] T. Ojala, M. Pietikainen and T. Maenpaa, "Multiresolution gray-scale and rotation invariant texture classification with local binary patterns," IEEE Transactions on Pattern Analysis and Machine Intelligence, vol. 24, no. 7, pp. 971-987, 2002.

[7] T. Ahonen, A. Hadid and M. Pietikinen, "Face recognition with local binary patterns," IEEE Conference of European Conference on Computer Vision, Prague, pp. 469-481, 2004.

[8] L. O. Chua, "CNN: a version of complexity," International Journal of Bifurcation and Chaos, vol. 7, pp. 2219-2425, 1997.

[9] H. Zhang, Z. Mu and C. Zhang, "Ear recognition method based on Independent Component Analysis and BP neural network," Journal of University of Science and Technology Beijing, vol. 28, no. 6, pp. 600-603, 2006.

[10] L. Yuan, Z. Mu, Y. Zhang and K. Liu, "Ear Recognition using Improved Non-Negative Matrix Factorization," The 18th International Conference on Pattern Recognition, Hong Kong, vol. 4, pp. 501-504, 2006. 